\title{
Explicit dynamic with X-FEM to handle complex geometries
}

\author{
P. Rozycki ${ }^{1}$, E. Bechet ${ }^{2}$, N. Moës ${ }^{1}$ \\ ${ }^{1}$ Institut de Recherche en Génie Civil et Mécanique UMR CNRS 6183 \\ Ecole Centrale de Nantes, 1 rue de la Noë, BP 92101, F-44321 Nantes Cedex 3 \\ patrick.rozycki@ec-nantes.fr; nicolas.moes@ec-nantes.fr \\ ${ }^{2}$ Laboratoire de Physique et Mécanique des Matériaux UMR CNRS 7554 \\ Université de Metz, Ile de Saulcy, F-57045 Metz Cedex 1 \\ bechet@univ-metz.fr
}

\begin{abstract}
Although the calculation capacities have considerably increased these last years, the complexity of the numerical simulations in dynamic fields still induce many problems, essentially due to CPU time calculation. For instance, the use of explicit scheme yields a critical time step. It depends on the greatest structure eigenvalue [1], [2]. Commonly, rather than to identify this eigenvalue, an upper approximation computed corresponds to the smaller characteristic size (if all elements share the same behavior). These critical time steps are usually induced by mesh constraints. For complex geometries, very small sized elements may arise. One approach is then to optimize the mesh by removing elements or by using mass scaling to improve the critical time step.

The work is based on the developments suggested for static problems, which are using the eXtended Finite Element Method [3], [4]. Thanks to the unity partition theory, it is possible to add some specific functions of enrichment to the conventional approximation field of the displacement. These added functions allow, for example, the treatment of cracks, material interfaces, holes, etc. Consequently, this approach authorizes the non-conformity between mesh and discontinuities.

This paper presents the work carried out about the X-FEM finite elements, which are dedicated to the dynamic explicit problems including holes or external surfaces. In a first part, the developments are presented for $1 \mathrm{D}$ cases and for mono-material structures. The objective is to propose the theoretical framework of the X-FEM finite element: a reformulation of the stiffness matrix and an adapted mass matrix offer the possibility to increase time step calculation in the case of non-meshed surfaces. A generalization of the method is then proposed for the 2D and 3D cases. Validations for different type of structure are exposed. The results in comparison with ABAQUS software are relevant and allows us to present some outlooks.
\end{abstract}

\section{References}

[1] M.N. Newmark, A method of computation for structural dynamics, Proc. ASCE 85, EM3, 1959.

[2] T. Belytschko, T.J.R. Hughes, Computational methods for transient analysis, North-Holland, 1986.

[3] N. Sukumar, D. L. Chopp, N. Moës and T. Belytschko, Modeling Holes and Inclusions by Level Sets in the Extended Finite-Element Method, Computer Methods in Applied Mechanics and Engineering, Vol. 190, Number 46-47, pp. 6183-6200, 2001

[4] C. Daux, N. Moës, J. Dolbow, N. Sukumar, and T. Belytschko, Arbitrary branched and intersecting cracks with the eXtended Finite Element Method, International Journal for Numerical Methods in Engineering, 48:1741-1760, 2000. 